\title{
ALGEBRAIC EXTENSION OF NORMED ALGEBRAS
}

\section{RICHARD ARENS AND KENNETH HOFFMAN}

1. Introduction. Let $A$ be a commutative ring, and $a_{1}, \cdots, a_{n}$ some of its elements. Suppose we want to enlarge $A$ to a $\operatorname{ring} B$ so as to be sure of having at least one solution of

$$
1.1 \quad x^{n}+a_{1} x^{n-1}+\cdots+a_{n}=0
$$

in $B$. The classical method of field extension (forming the polynomial ring $A[x]$ and reducing modulo the principal ideal $J$ of 1.1) solves this problem. In this paper, we investigate the role of this process in topological rings.

We study the problem of obtaining a normed linear algebra extension $B$ containing a solution of 1.1 , where $A$ is a given normed linear algebra. Our method is to define a norm first in the polynomial algebra $A[x]$, and to do it in such a way that $J$ is closed (for otherwise the canonical norm in

$$
B=A[x] / J
$$

is not available) and that the imbedding of $A$ in 1.2 is an isometry.

If $A$ is complete (i.e., a Banach algebra), $B$ is likewise.

It will occur to some readers to ask if $B$ is semi-simple if $A$ is. The proper question is really this: if the intersection of the closed maximal ideals (we presuppose a unit in $A$ ) is (0), is the same true for $B$ ? The answer is in some cases, "no," but in those cases the discriminant of 1.1 is a zero divisor. But in any event, another ideal (perhaps (0)) can be divided out of $B$ leaving an algebra satisfying this requirement, as well as the others.

It seems difficult to treat all these questions (mainly the last one) when the leading coefficient of the polynomial has no inverse.

2. Commutativity assumed. In what follows, $A$ will stand for a linear algebra over the complex numbers $C$, with a norm with the usual properties, in particular $\|x y\| \leqq\|x\|\|y\|$.

This much is usually called a normed linear algebra (and if also complete in the norm, a Banach algebra).

In order to save space, we shall use the shorter phrase, "normed algebra," to mean even more: namely, a commutative normed linear algebra with unit element.

Received by the editors May 9, 1955. 
By an isometric extension $B$ of $A$ we mean a normed algebra with a subalgebra $B_{0}$ which is algebraically isomorphic and norm-isometric with $A$.

3. Algebraic extension. Let $a_{0}, a_{1}, \cdots, a_{n}(n>1)$ be elements of $A$. We want an extension $B$ of $A$ such that

$$
\alpha(x)=a_{0}+a_{1} x+\cdots+a_{n} x^{n}
$$

has a zero in $B$. We form the algebra $A[x]$ of all polynomials

$$
\gamma(x)=c_{0}+c_{1} x+\cdots+c_{p} x^{p}
$$

over $A$, and let

$$
B=A[x] /(\alpha(x))
$$

where $(\alpha(x))$ is the principal ideal of 3.1 in $A[x]$.

We need a norm in $B$. Our method is to define a norm in $A[x]$, and then use the canonical method (cf. Loomis $[3$, p. 14]) for norming the quotient algebra 3.3. (In general, this method merely "pseudo-norms" 3.3.) Of the many ways of norming $A[x]$, we present here a oneparameter family of norms. Select a positive $t$, and then for every element of $A[x]$, say 3.2 , let

$$
\|\gamma(x)\|=\left\|c_{0}\right\|+\left\|c_{1}\right\| t+\cdots+\left\|c_{p}\right\| t^{p} .
$$

No matter what $t$ is, the subalgebra of "constant" polynomials is isometric and isomorphic to $A$. Now we pass to the quotient algebra 3.3 , using the canonical norm in the latter. Our concern is to choose $t$ in such a way that the canonical homomorphism

$$
A[x] \rightarrow A[x] /(\alpha(x))
$$

shall be isometric as applied to the constant polynomials. The following is essentially our solution to this problem (see also 3.6 below).

3.5. Lemma. If the polynomial $\alpha(x)$ has the monic form

$$
\alpha(x)=a_{0}+a_{1} x+\cdots+a_{n-1} x^{n-1}+x^{n}
$$

and $t$ satisfies the condition

$$
\left\|a_{0}\right\|+\left\|a_{1}\right\| t+\cdots+\left\|a_{n-1}\right\| t^{n-1} \leqq t^{n},
$$

then the ideal $(\alpha(x))$ is closed in $A[x]$, and the natural image of $A$ in $B$ (see 3.3) is isometrically isomorphic to $A$. Moreover, if $A$ is complete, so is the extension $B$.

Proof. It is no real loss of generality to suppose that $t=1$ : 


$$
\left\|a_{0}\right\|+\left\|a_{1}\right\|+\cdots+\left\|a_{n-1}\right\| \leqq 1 .
$$

Then 3.4 is simply

$$
\|\gamma(x)\|=\left\|c_{0}\right\|+\cdots+\left\|c_{p}\right\| .
$$

Each element $\delta(x)$ in $A[x]$ is equivalent, modulo $\alpha(x)$, to a unique polynomial of the form

$$
\beta(x)=b_{0}+b_{1} x+\cdots+b_{n-1} x^{n-1} .
$$

The norm of (the coset of) $\delta(x)$, in the quotient ring $B$, will be

$$
N(\delta)=\inf _{\gamma}\|\delta(x)+\alpha(x) \gamma(x)\| .
$$

In the right hand of $3.54, \delta$ may be replaced by $\beta$. (It is not yet evident that $N(\cdot)$ is a norm, because it has yet to be seen that $N(\delta) \neq 0$ if $\delta$ does not lie in the ideal $(\alpha(x))$. We exhibit 3.54 here merely as a motivation.) We wish to prove that

3.55

$$
D=\|\beta(x)+\alpha(x) \gamma(x)\| \geqq\|\beta(x)\|
$$

(and then it will result that $N(\delta)=\|\beta(x)\|)$. We suppose $\gamma(x)$ has the form 3.2. By 3.52,

$$
\begin{aligned}
D= & \left\|b_{0}+a_{0} c_{0}\right\|+\left\|b_{1}+a_{0} c_{1}+a_{1} c_{0}\right\|+\cdots \\
& +\left\|b_{n-1}+a_{0} c_{n-1}+\cdots+a_{n-1} c_{0}\right\| \\
& +\left\|a_{0} c_{n}+a_{1} c_{n-1}+\cdots+a_{n-1} c_{1}+c_{0}\right\| \\
& +\left\|a_{0} c_{n+1}+\cdots+a_{n-1} c_{2}+c_{1}\right\|+\cdots .
\end{aligned}
$$

The $c_{i}$ stand alone in the terms of the second type because $a_{n}=1$. For visual ease, we shall, in the next few lines, denote the norm of $a_{m}, b_{m}$, $c_{m}$ by capital letters of the same type and with the same subscript. By the norm properties of normed algebras, we get

$$
\begin{aligned}
D \geqq & \left(B_{0}-A_{0} C_{0}\right)+\left(B_{1}-A_{0} C_{1}-A_{1} C_{0}\right)+\cdots \\
& +\left(B_{n-1}-A_{0} C_{n-1}-\cdots-A_{n-1} C_{0}\right) \\
& +\left(C_{0}-A_{0} C_{m}-A_{1} C_{n-1}-\cdots-A_{n-1} C_{1}\right) \\
& +\left(C_{1}-A_{0} C_{n-1}-\cdots-A_{n-1} C_{2}\right)+\cdots \\
= & B_{0}+B_{1}+\cdots+B_{n-1} \\
& +C_{0}\left(1-A_{0}-A_{1}-\cdots-A_{n-1}\right) \\
& +C_{1}\left(1-A_{0}-A_{1}-\cdots-A_{n-1}\right) \\
& +C_{2}\left(1-A_{0}-A_{1}-\cdots-A_{n-1}\right)+\cdots
\end{aligned}
$$

By $3.52,\|\beta(x)\|=B_{0}+\cdots+B_{n-1}$, while by $3.511,1-A_{0}-\cdots$ 
$-A_{n-1} \geqq 0$. These three relations yield 3.55 . Now 3.55 yields

$$
N(\delta) \geqq\|\beta(x)\| .
$$

Of course, the opposite inequality also holds. Thus

$$
N(\delta)=\|\beta(x)\| .
$$

This means that the norm in $B$ can be found for each coset $\delta(x)$ $+(\alpha(x))$ by selecting the unique element 3.53 from that coset and calculating its norm. Thus $B$ is isometric with the algebra

$$
A \times A \times \cdots \times A
$$

with the norm

$$
\left\|\left(b_{0}, b_{1}, \cdots, b_{n-1}\right)\right\|=\left\|b_{0}\right\|+\left\|b_{1}\right\|+\cdots+\left\|b_{n-1}\right\|
$$

(and a product obtained by regarding the elements as polynomials of degree less than $n$, and replacing $x^{n}$, when it arises, by $-a_{0}-a_{1} x-\ldots$ $\left.-a_{n-1} x^{n}\right)$. It follows first, that the ideal $(\alpha(x))$ is closed and, second, that $B$ is complete when $A$ is. This completes the proof of 3.5 .

If in the equation

$$
a_{0}+a_{1} x+\cdots+a_{n} x^{n}=0,
$$

to be solved in the extension, the coefficient $a_{n}$ has an inverse, then the equation can be immediately replaced by one in which this coefficient is 1 . For this, a $t$ satisfying 3.51 can be found. The following sums up the results.

3.6. THEOREM. Let the leading coefficient $a_{n}$ of $\alpha(x)$ have an inverse. Then a norm of type 3.4 can be found for $A[x]$ such that the algebra $B$ (see 3.3) (in which $\alpha(x)$ has a root) is an isometric extension of $A$, and is complete when $A$ is complete.

4. Maximal ideals. In a topological ring, the closed ideals are of greater interest than the others. Our attention is presently brought to the class of closed regular maximal right (or left) ideals, and to the intersection of that class (which when the word "closed" is omitted, is called the radical by Jacobson [2]). This closed ideal contains Jacobson's radical, and may be large even when the latter is trivial. Perhaps it might be called the refractory ideal. In any case, we need an adjective signifying that it reduces to $(0)$ for a given ring. Rather than "topologically semi-simple," we shall use "tractable." A tractable topological ring is therefore semisimple, but not conversely.

A normed algebra $A$ satisfying the conditions of $\$ 2$ is (therefore) tractable if the intersection of all closed maximal ideals is merely (0). 
If $M$ is one of these maximal closed ideals, then $A / M$ with the canonical norm is isomorphic and isometric with the complex numbers $C$, as stated by Mazur (see Arens $[1$, p. 626]). $M$ is the kernel of a continuous (or, what is the same, bounded) homomorphism $h$ of $A$ onto $C$. The class of these we shall denote by $H(A)$. As with Banach algebras, $H(A)$ is a subset of the conjugate space $A^{-}$, and in the weak topology, is compact.

We return to the algebra $A[x]$.

4.1. Theorem. If $A[x]$ has norm 3.52 ,

$$
H(A[x])=H(A) \times\{\lambda ;|\lambda| \leqq 1\} .
$$

Moreover, $A[x]$ is tractable (see above) precisely when $A$ is.

Indeed, for any $(h, \lambda)$ from the right side of 4.11 , and any $\lambda(x)$ (see 3.2), we can define a member of the left side by setting

$$
(h, \lambda)(\gamma(x))=h\left(c_{0}\right)+\lambda h\left(c_{1}\right)+\cdots+\lambda^{p} h\left(c_{p}\right)=h(\gamma(\lambda)) .
$$

4.2. Theorem. If $A[x]$ has norm 3.52 and 3.511 holds, then $H(B)$ is that part of $H(A[x])$ on which

$$
(h, \lambda)(\alpha(x))=0 .
$$

This proposition follows from the fact that the ideal generated by $\alpha(x)$ is closed.

As the discriminant of a polynomial $\alpha(x)$ in an indeterminate $x$ with coefficients in a commutative ring we can take the resultant of $\alpha(x)$ and $\alpha^{\prime}(x)$, removing therefrom the leading coefficient which is formally a factor of every term, to agree with classical usage (cf. [4, p. 93]). Then the discriminant lies in the ring, provided the latter has an identity element.

4.3. Theorem. Under the hypotheses of the Lemma 3.5, if $A$ is tractable and the discriminant, $d$, of $\alpha(x)$ is not a zero divisor, then $B$ is tractable.

Proof. We may again assume 3.511, with no loss of generality. Suppose that $\beta(x)$ (3.53) lies in every closed maximal ideal of $B$. Then according to Theorem 4.2 , for every $(h, \lambda)$ such that 4.21 holds,

$$
\lambda^{n-1} h\left(b_{n-1}\right)+\cdots+\lambda h\left(b_{1}\right)+h\left(b_{0}\right)=0 .
$$

Let $h$ be any element of $H(A)$ for which $h(d) \neq 0$. If we regard 4.21 as a polynomial equation with unknown $\lambda$, the equation has the discriminant $h(d)$ and, hence, has $n$ distinct complex roots. Each of these roots must also satisfy 4.31 , which is of degree less than $n$. It follows that $h\left(b_{k}\right)=0$, for $k=0,1, \cdots, n-1$. 
Therefore, for any $h$ in $H(A), h\left(d \cdot b_{k}\right)=0$. Since $A$ is tractable, each $d \cdot b_{k}$ is zero. But, as $d$ is not a zero divisor, this implies $\beta(x)=0$.

4.4. Corollary. If $A$ is tractable and

$$
\alpha(x)=x^{n}+a_{0}
$$

then a necessary and sufficient condition that $B$ be tractable is that $a_{0}$ not be a zero divisor.

Proof. The sufficiency follows from the theorem. Suppose there is a nonzero element $a$ in $A$ such that $a \cdot a_{0}=0$. Then the element $a x$ lies in every closed maximal ideal of $B$. This may be seen as follows: From Theorem 4.2, the elements of $H(B)$ are the homomorphisms $(h, \lambda)$, where

$$
(h, \lambda)(a x)=\lambda \cdot h(a)
$$

and $\lambda^{n}=-h\left(a_{0}\right)$. But, for any $h$ in $H(A), h(a) h\left(a_{0}\right)=0$. Hence, $(h, \lambda)(a x)=0$. Therefore, $B$ is not tractable.

In the event that $B$ is not tractable (when $A$ is), we can still determine (for certain $\alpha(x)$ ) a tractable isometric extension of $A$ in which $\alpha(x)=0$ has a root.

We introduce the following notation. For each $k=0,1, \cdots, n-1$, let $q_{k}=q_{k}\left(a_{0}, \cdots, a_{n-1}\right)$ be the sum of the $k$ th powers of the roots of the equation

$$
\alpha(x)=a_{0}+a_{1} x+\cdots+a_{n-1} x^{n-1}+x^{n}=0 .
$$

Then $q_{k}$ is an element of $A$, and can be expressed as a polynomial in the coefficients of $\alpha(x)[4$, p. 86, exercise 4]. Now let $K(B)$ be the intersection of the closed maximal ideals of $B$. Then we have the following:

4.5. Lemma. Under the hypotheses of Lemma 3.5, if $A$ is tractable and if the number $t$ also satisfies the conditions

$$
\left\|q_{k}\right\| \leqq t^{k}, \quad k=1, \cdots, n-1,
$$

then the canonical mapping

$$
B \rightarrow B / K(B)
$$

restricted to $A$ is an isometry.

Proof. No generality is lost if we assume 3.511 and

$$
\left\|q_{k}\right\| \leqq 1 \quad(k=1, \cdots, n-1) .
$$

Let $\beta(x)$ (3.53) be in $K(B)$. Let $h$ be any element of $H(A)$, and let 
$\lambda_{1}, \cdots, \lambda_{n}$ be the (not necessarily distinct) roots of 4.21 . According to 4.2 we have, for $j=1,2, \cdots, n$,

$$
\lambda_{i}^{n-1} h\left(b_{n-1}\right)+\cdots+\lambda_{j} h\left(b_{1}\right)+h\left(b_{0}\right)=0 .
$$

If we add the $n$ equations 4.52 , we obtain

$$
\sum_{k=0}^{n-1}\left(\sum_{j=1}^{n} \lambda_{j}^{k}\right) h\left(b_{k}\right)=0 .
$$

But,

$$
\sum_{j=1}^{n} \lambda_{j}^{k}=h\left(q_{k}\right) .
$$

Then 4.53 becomes (recalling that $h$ is multiplicative)

$$
\sum_{k=0}^{n-1} h\left(q_{k} b_{k}\right)=0 \text {. }
$$

Since $A$ is tractable, and $h$ was arbitrary,

$$
\sum_{k=0}^{n-1} q_{k} b_{k}=0
$$

Now $q_{0}=n$, so that 4.54 can be rewritten $b_{0}=-(1 / n) \sum_{k=1}^{n-1} q_{k} b_{k}$.

From the norm properties of a normed algebra and from 4.51, we obtain

$$
\left\|b_{0}\right\| \leqq \sum_{k=1}^{n-1}\left\|b_{k}\right\|\left\|q_{k}\right\| \leqq \sum_{k=1}^{n-1}\left\|b_{k}\right\|
$$

Now, let $a$ be an element of $A$. From 3.57,

$$
\begin{aligned}
\|\beta(x)-a\| & =\left\|b_{0}-a\right\|+\left\|b_{1}\right\|+\cdots+\left\|b_{n-1}\right\| \\
& \geqq\|a\|-\left\|b_{0}\right\|+\left\|b_{1}\right\|+\cdots+\left\|b_{n-1}\right\| .
\end{aligned}
$$

Applying 4.55, we obtain

4.56

$$
\|\beta(x)-a\| \geqq\|a\| \text {. }
$$

Under the mapping $B \rightarrow B / K(B)$, the coset of an element $a$ in $A$ (regarded as an element of $B$ ) will have the norm

$$
M(a)=\inf \|\beta(x)-a\|, \quad \beta(x) \text { in } K(B) .
$$

Certainly $M(a) \leqq\|a\|$; whereas, 4.56 yields the reverse inequality. Therefore, $M(a)=\|a\|$. This completes the proof of 4.5.

Since the conditions imposed on $t$ can easily be fulfilled in any 
given case, we can assert the following result on algebraic extension.

4.6. ThEOREM. Let $A$ be a tractable normed [Banach] algebra. Let $\alpha(x)$ (3.1) be a polynomial over $A$ for which $a_{n}$ has an inverse. Then there exists a tractable normed [Banach] algebra, which is an isometric extension of $A$, and in which the equation $\alpha(x)=0$ has a root.

\section{BIBLIOGRAPHY}

1. R. Arens, Linear topological division algebras, Bull. Amer. Math. Soc. vol. 53 (1947) pp. 623-630.

2. N. Jacobson, The radical and semi-simplicity for arbitrary rings, Amer. J. Math. vol. 67 (1945) pp. 300-320.

3. L. Loomis, An introduction to abstract harmonic analysis, New York, van Nostrand, 1953.

4. B. L. Van der Waerden, Moderne Algebra, Berlin, 1937.

University of California, Los Angeles 